\title{
Research on military electrical and electronic products based on the influence of biological environment.
}

\author{
Zhang Jianming* \\ Weifang University of Science and Technology, Weifang, Shandong, PR China
}

\begin{abstract}
The biological environment condition has a very important influence on the effective preservation of military electrical and electronic products. In this paper, the research on military electrical and electronic products based on the influence of biological environment was carried out, and the main contents of the biological environment were introduced. At the same time, the influence factors and research methods of biological environment on military electrical and electronic products were expounded and the research results were analysed and discussed. The results show that the adverse effects of mold on military electrical and electronic products are high under the condition of high temperature. The author believes that the main method to reduce the adverse effects of biological environment on military electrical and electronic products is to control the storage temperature.
\end{abstract}

Keywords: Biological environment, Military, Electronic products.

Accepted on June 19, 2017

\section{Introduction}

The storage environment of military electrical and electronic products contains a large number of microorganisms. These microorganisms are dominated by various molds and fungus. The military electrical and electronic products are easily affected by the biological environment, leading to corrosion and damage [1]. The biological environmental conditions include climatic and environmental conditions. Under the condition of wet tropical climate, biological and environmental conditions have much effect on the military electrical and electronic products. The biological environment mainly includes microorganisms, insects and rodents. The most harmful effect on the military electrical and electronic products is the mold. This is mainly because the mold will damage the military electrical and electronic products. The metabolic process of mold can damage the circuit of products [2]. Rodents, such as rats can gnaw the shell of the military electrical and electronic products. Once the appearance of the military electrical and electronic products is destroyed, microbes can easily cause corrosive damage to electronic circuits. Therefore, on the basis of the relevant theories at home and abroad, this paper analyses and studies the subject of the military electrical and electronic products based on the influence of biological environment.

\section{State of the Art}

The factor analysis method is also called the index factor analysis method; it is a statistical method to analyse the degree of each factor in the total variation of the phenomenon by using the statistical index system, including the chain substitution method, the difference analysis method, the index decomposition method, the fixed base substitution method. Factor analysis method is an important and practical method in modern statistics, and it is a branch of multivariate statistical analysis. Factor analysis method can be used to find out the influence factors that can be used to measure the research object according to the actual situation, so as to get the results through the analysis of the relationship and characteristics of the factors by the use of the mathematical calculation. It can be seen that the factor analysis method can simplify the analysis of the factors which have a certain degree of complexity and optimize the research methods.

\section{Methodology}

Mold is the most important component of the military electrical and electronic products in biological environment. This paper mainly analyses and studies the relationship between the biological environment conditions and the military electrical and electronic products. The adverse effects of mold in storage environment on biological conditions are mainly reflected in three aspects: temperature, humidity and nutrients. Temperature directly affects the survival of mold. Rising temperature can promote the growth of mold. Humidity is a necessary condition for mold. This is mainly because mold will stop growing and even die if there are not enough humidity environments. The main nutrients in the growth and development of mold include carbon, nitrogen and inorganic salts. These nutrients are important nutrients to help mold survive. Lack of nutrients will seriously affect the growth of mold. Thus, temperature, humidity and nutrients have a decisive impact on the growth and development of mold. The 
adverse effects of environmental conditions on the military electrical and electronic products can be reduced by controlling the temperature, humidity and nutrients.

$\sigma=\sqrt{\frac{\sum(x-\bar{x})^{2} f}{\sum f}} \rightarrow$

$\mu=\sqrt{\frac{\sigma^{2}\left(1-\frac{n}{N}\right)}{n}} \rightarrow(2)$

The relationship between the biological environment conditions and the military electrical and electronic products was analysed by using the factor analysis method: First of all, the investigation of the biological environment of the military electrical and electronic products was carried out; then, according to the information obtained from the survey, the formula (1) was used to determine the influencing factors; finally, the formula (2) was used to calculate and analyse the relationship between the factors. Factor analysis method has high objectivity and scientificity. The accuracy of the relationship between the biological environment conditions and the military electrical and electronic products can be ensured by using the factor analysis method, at the same time, a more comprehensive analysis of the subject can be made, so as to find out the effective ways to reduce the adverse effects of the biological environment on the military electrical and electronic products.

\section{Result Analysis and Discussion}

The results obtained by the method described above are shown in the following tables:

Table 1. Analysis of influencing factor index.

\begin{tabular}{lll}
\hline Factor & Standard index & Influence index \\
\hline Temperature & 0.6 & 0.89 \\
\hline Humidity & 1 & 0.67 \\
\hline Nutrients & 0.8 & 0.54 \\
\hline
\end{tabular}

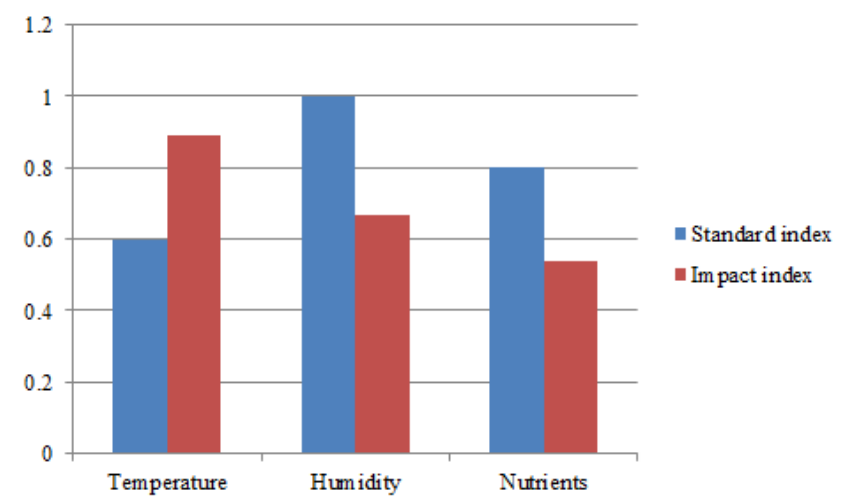

Figure 1. Comparison and analysis of the influence of mold on the military electrical and electronic products.

From Table 1 and Figure 1, the data show that the harmful effect of mold on the military electrical and electronic products is high under the condition of high temperature. This is mainly because the temperature is too high and reaches the appropriate growth temperature, mold growth rate will continue to accelerate, and lead to a sharp increase in the number of mold. When the humidity and nutrient index exceed the standard index, which is conducive to the survival of the mold, however, the influence of these two factors on military electrical and electronic products is lower than that of temperature. Therefore, the author puts forward effective strategies to minimize the adverse effects of biological environment on military electronic products. Firstly, the temperature level of the storage environment of the military electrical and electronic products needs to be monitored in real time, so as to avoid the suitable temperature range of mold. Secondly, it is necessary to maintain the storage environment dry. Finally, the storage environment needs to be cleaned up in real time, so as to effectively reduce the level of nutrients. It can be seen that the effective control of the biological environment can effectively reduce the damage to the military electrical and electronic products.

\section{Conclusions}

The effective preservation of the military electrical and electronic products is very important. Therefore, it is very important to research the military electrical and electronic products based on the influence of biological environment. Based on the factors analysis method, this paper studied the military electrical and electronic products based on the influence of biological environment, expounded the influence factors and research methods of biological environment on military electrical and electronic products, and summarized the relationship between the biological environment conditions and the military electrical and electronic products by analyzing the data, so as to provide some reference basis for avoiding the adverse effects of mold. In the author's opinion, the harmful effect of mold on the military electrical and electronic products is high under the condition of high temperature. The effective control of the biological environment can effectively reduce the damage to the military electrical and electronic products.

\section{References}

1. Wang JP, Office MR, Factory DI. Analysis of Environment Stress Screen (ESS) for the electronics products' of torpedo. Environ Technol 2015.

2. Padmavathiamma PK, Ahmed M, Rahman HA. Phytoremediation a sustainable approach for contaminant remediation in arid and semi-arid regions-a review. Emi J Food Agr 2014; 26: 458-474.

\section{*Correspondence to}

Zhang Jianming

Weifang University of Science and Technology

Shandong

PR China 